\title{
Approach to Meniscal Tear by Clinical Examination and Its Management: A Review
}

\author{
Roshan Sah ${ }^{1}$, Luike Bin'1, Vijay Kumar Sah"1, Keshav Singh Dhami2 \\ ${ }^{1}$ Department of Orthopedics, The First Affiliated Peoples Hospital of Yangtze University, Jingzhou, China \\ ${ }^{2}$ Jingzhou Central Hospital, Second Clinical Medical Hospital, Yangtze University, Jingzhou, China \\ Email: ^dr.roshan916@outlook.com
}

How to cite this paper: Sah, R., Bin, L.K., Sah, V.K. and Dhami, K.S. (2019) Approach to Meniscal Tear by Clinical Examination and Its Management: A Review. Yangtze Medicine, 3, 195-211.

https://doi.org/10.4236/ym.2019.33020

Received: November 28, 2018

Accepted: Jun. 25, 2019

Published: Jun. 28, 2019

Copyright $\odot 2019$ by author(s) and Scientific Research Publishing Inc. This work is licensed under the Creative Commons Attribution International License (CC BY 4.0).

http://creativecommons.org/licenses/by/4.0/ (c) (i) Open Access

\begin{abstract}
It's quite difficult to diagnose the meniscus tear by clinical examination even the experienced orthopaedic surgeon, so this review has shortly focused on clinical examination and its treatment. How to overcome missed diagnosis of meniscus tear in clinical setting? This review article highlighted the importance of early diagnosis and treatment; fortunately, these processes have been vastly improved by advances in Magnetic resonance imaging (MRI) and Arthroscopy. Although partial, subtotal and total meniscectomy is the choice for different types of meniscal tears, there are certain criteria to be meet for undergoing surgical treatment. The most common criteria for meniscal repair include: 1) A vertical longitudinal tear more than $1 \mathrm{~cm}$ in length located within vascular zone, 2) A tear which is unstable and displaceable into the joint, 3) An active informed and cooperative patient but younger than 40 years old, 4) A stable knee or wound stabilized with a ligamentous reconstruction. Chronically deformed or degenerative menisci are bottom-out candidates for repair. Most investigators report that only $10 \%$ to $15 \%$ of meniscal tears can be repaired and that most such repairs are done in association with an anterior cruciate ligament reconstruction. However, this article presents a review of the clinical relevant anatomic, function, repair and healing mechanism, description of the meniscus attachment as well as current strategies for accurate diagnosis and treatment of common injuries to these meniscus attachments.
\end{abstract}

\section{Keywords}

Meniscus, Healing and Repair, Medial Meniscus, Lateral Meniscus, Diagnostic and Clinical Test

\section{Introduction}

Meniscal function is essential to the normal function of the knee joint. For the 
menisci to function properly, the biomechanical integrity of each meniscus root on the tibial plateau must be maintained [1] [2]. Various functions have been attributed to the menisci, some of which are known or proved and others that are theorized. The menisci act as joint filler, compensating for gross incongruity between femoral and tibial articulating surface. So located, the menisci prevent capsular and synovial impingement during flexion-extension movements. The menisci have been believed to have a joint lubrication function, helping to distribute synovial fluid throughout the joint and aiding the nutrition of the articular cartilage [3] [4] [5] [6]. They undoubtedly contribute to stability in all planes but are especially important rotary stabilizers and are probably essential for the smooth transition from a pure hinge to a gliding or rotary motion as the knee moves from flexion to extension. The menisci are crescents, roughly triangular in cross-section, that cover one half to two-thirds of the articular surface of the corresponding tibial plateau [7] [8] [9]. They are composed of dense, tightly woven collagen fibres arranged in a pattern providing great elasticity and ability to withstand compression. The major orientation of collagen fibres in the meniscus is circumferential, radial fibres and perforating fibres determines to some extent the characteristic pattern of meniscal tears. When meniscal samples are tested by application of a force perpendicular to the fibre direction, the strength is decreased to less than $10 \%$ because collagen fibres function primarily to resist tensile force along the direction of the fibres. The circumferential fibres act much in the same way as metal hoops are placed around a pressurized wooden barrel [3] [4] [6].

The peripheral edges of menisci are convex [3] [10], fixed and attached to the inner surface of the knee joint capsule, except where the popliteus is interposed laterally; these peripheral edges also are attached loosely to the borders of the tibial plateaus by the coronary ligaments. The inner edges are concave, thin and unattached. The menisci are largely a vascular except near their peripheral attachment to the coronary ligament. The inferior surface of each meniscus is flat, contour of the underlying tibial plateau and superimposed femoral condyle. The medial meniscus is a C-shaped structure larger in radius than the lateral meniscus, with the posterior horn being wider than the anterior. The anterior horn is attached firmly to the tibia anterior to the intercondylar eminence and to the anterior cruciate ligament. Berlet and Flowler also described the anterior horn as associating with the ACL in 59\% of knees. Other researchers have reported the anterior horn as connecting to the anterior intermeniscal ligament, also known as transverse ligament, in approximately $70 \%$ of knees [8] [11] [12] [13]. Most of the weight is borne on the posterior portion of the meniscus. The posterior horn is anchored immediately in front of the attachments of the eminence. Its entire peripheral border is firmly attached to the medial capsule and through the coronary ligament to the upper border as the tibia. The Lateral meniscus is smaller in diameter, thicker in periphery, wider in body, and more than the medial meniscus. It is attached to both cruciate ligaments and posteriorly to the medial femoral condyle by either the ligament of Humphry or the ligament of Wrisberg, de- 
pending on which is present; it is also attached posteriorly to the popliteus muscles. It is separated from the lateral collateral ligament by the popliteal tendon. In contrast, the medial meniscus is much larger in diameter, is thinner in its periphery and narrower in body, and does not attached to either cruciate ligament. It is loosely attached to the medial capsular ligaments. Zantop reported that anteromedial bundle of the ACL was an average of $5.2 \mathrm{~mm}$ medial and $2.7 \mathrm{~mm}$ posterior to the lateral anterior root, while the poster lateral bundle was an average $11.2 \mathrm{~mm}$ posterior and $4.1 \mathrm{~mm}$ medial to the anterior root [14]. Ziergler reported that centre of ACL was on average $7.5 \mathrm{~mm}$ medial to the anterior root of the lateral meniscus centre, $8.5 \mathrm{~mm}$ anteromedial to the poster medial aspect of the anterior horn of the lateral meniscus and 10.2 poster medial to the anteromedial aspect of the anterior horn [15]. The vascular supply to the medial and lateral menisci originates predominantly from the lateral and medial geniculate vessels (both inferior and superior). Branches from these vessels give rise to a perimeniscal capillary plexus within the synovial and capsular tissue.

\section{Meniscal Healing and Repair}

The vascular supply to the meniscus determines its potential for repair. The peripheral meniscal blood supply is capable of producing a reparative response similar to that observed in other connective tissues because of a perimeniscal capillary plexus that supplies the peripheral $10 \%$ to $25 \%$ of the menisci. Meniscal tears have been classified based on their location in three zones of vascularity

1) Red (fully within the vascular area).

2) Red-white (at the border of the vascular area).

3) White (within the a vascular area).

This classification indicates the potential for healing after repair and section on surgical repair of torn menisci. After injury within the peripheral vascular zone, a fibrin clot that is rich in inflammatory cells forms. The healing process depend on debriment or abrasion of wall of the tear, especially in chronic lesion [16]. Vessels from the peri-meniscal capillary plexus proliferate throughout this fibrin scaffold and are accompanied by the proliferations of differentiated mesenchymal cells. The lesion is eventually filled with cellular fibro vascular scar tissue that glues the wound edges together and appears continuous with the adjacent normal meniscal fibro cartilage. Vessels from the peri-meniscal capillary plexus as well as the proliferative vascular pannus from the synovial fringe penetrate the fibrous scar to provide a marked inflammatory response. Although several months are required for maturation to fibro cartilage that appears normal.

\section{Tears of Menisci}

A rotational force incurred while the joint is partially flexed usually tears a meniscus. During vigorous internal rotation of the femur on the tibia with the knee flexion, the femur tends to force the medial meniscus posteriorly and toward the centre of the joint. A strong peripheral attachment posteriorly may prevent the 
meniscus from being injured, but if this attachment stretches or tears, the posterior part of the meniscus is forced toward the centre of the joint, is caught between the femur and the tibia, and is torn longitudinally when the joint is suddenly extended. If this longitudinal tear extends anteriorly beyond the medial collateral ligament, the inner segment of the meniscus is caught in the intercondylar notch and cannot to its former position: thus, a classic bucket-handle tear with locking of the joint is produced. The same mechanism can produce a posterior peripheral or a longitudinal tear of the lateral meniscus; the lateral femoral condyle forces the anterior half of the meniscus anteriorly and toward the centre of the joint, and this strain in turn may tear the posterior half of the meniscus from its peripheral attachment. When the joint is extended, a longitudinal tear results. Because it not as susceptible to bucket-handle tears; however, because it is more sharply curved and is neither attached to nor controlled by the lateral collateral ligament, the lateral meniscus sustains incomplete transverse tears more often than does the medial meniscus.

\section{Classification}

Numerous classifications of tears of the menisci have been proposed on the basis of location or type of tear, etiology and other factors; most of the commonly used classification are based on the type of tear found at surgery:

1) Longitudinal tears.

3) Transverse and oblique tears.

3) Combination of longitudinal and transverse tears.

4) Tears associated with cystic menisci.

5) Tears associated with discoid menisci.

The most common type of tear is longitudinal tear, usually involving the posterior segment of either the medial or the lateral meniscus, before the extensive use of arthroscopy for diagnosis and treatment of meniscal injuries, tears of the medial meniscus in most series were approximately five to seven times more common than those of the lateral meniscus. However, increase in use of the arthroscopy, allow better inspection of both menisci, more lateral meniscal tears have been diagnosed. Small tears limited to the posterior horn are not capable of producing locking but will cause pain, recurrent swelling and a feeling of instability in the joint. Extensive longitudinal tear can cause mechanical locking if the central portion of the meniscus is displaced into the intercondylar notch. A pedunculated fragment may result if either the posterior or the anterior attachment of the bucket-handle fragment becomes detached. Transverse, radial or oblique tears can occur in either meniscus but more commonly involve the lateral meniscus. Because the lateral meniscus is more of a circle and has a shorter radius, the inner free edge transversely torn more easily than its medial counter-part. Transverse tears also can result from degenerative change within the meniscus itself or from injury or conditions such as cystic changes at the periphery that render the meniscus less mobile. Cysts of menisci are frequently associated with tears and are nine times more common on the lateral than on the medial side. 
The most common cause is trauma that produces degeneration and secondary mucinous and cystic changes follow, the meniscus may become less mobile during flexion, extension and rotary motions and thus more susceptible to additional longitudinal or transverse tearing. Discoid menisci are abnormal and because of hyperactive mobility and the bulk of the tissue between the articular surfaces, they are vulnerable to compression and rotary stresses, may develop degeneration within the discoid meniscus, as well as tear. The diagnosis often is not produce significant symptoms unit some derangement of the meniscus occurs.

\section{Diagnosis}

The diagnosis of a meniscal tear can be difficult even for an experienced orthopaedic surgeon. Use of a careful history and physical examination and supplementation of standard radiographs in specific instances with special imaging techniques and arthroscopy can keep errors in diagnosis of tears of the menisci to less than $5 \%$. When a meniscus has been injured, capsular and ligamentous structures as well as the articular surfaces also often have been injured. The syndrome caused by tears of the menisci can be divided into two group; those in which there is locking is absent and the diagnosis is more difficult. If a patient does not have locking, the diagnosis of a torn meniscus is more difficult even for the most astute surgeon. A patient typically gives a history of several episodes of trouble referable to the knee, often resulting in effusion and a brief period of disability but no definite locking. A sensation of "giving way" or snaps, clicks, catches, jerks in the knee may be described, or the history may be even more indefinite, with recurrent episodes of pain and mild effusion in the knee and tender seen in the anterior joint space after excessive activity. When they are well understood, the following clues can be important in the differential diagnosis in this second group: a sensation of giving way, effusion, atrophy of the quadriceps, tenderness over the joint line (or the meniscus during the physical examination).

A Sensation of giving way is in itself of little help in diagnosis because it can occur in other disturbances of the knee, especially loose bodies, chondromalacia of the patella and instability of the joint resulting from injury to the ligaments or from weakness of the supporting musculature, especially the quadriceps. When this symptom results from a tear in the posterior part of a meniscus, the patient usually notices this on rotary movements of the knee and often associates it with a feeling of subluxation or "the joint jumping out of place". When giving way is a result of other cause, such as quadriceps weakness, it usually is noticeable during simple flexion of the knee against resistance, such as in walking down stairs.

\section{Diagnostic Clinical Tests}

\subsection{The McMurray Test}

With the patient supine and the knee acutely and forcibly flexed, the examiner can check the medial meniscus by palpating the poster medial margin of the 
joint with one hand while grasping the foot with the other hand. Keeping the knee completely flexed, the leg is externally rotated to the maximum extent and then the knee is slowly extended. As the femur passes over a tear in the meniscus, a click may be heard or felt. The lateral meniscus is checked by palpating the poster lateral margin of the joint, internally rotating the leg as far as possible and slowly extending the knee while listening and feeling for a click. A click produced by the McMurray test is usually caused by a posterior peripheral tear of the meniscus and occurs between complete flexion of the knee to 90 degrees. Popping, which occurs with greater degrees of extension when it is definitely localized to the joint line, suggests a tear of the middle and anterior portions of the meniscus. The position of the knee when the clicks occur thus may help locate the lesion. A McMurray click localized to the joint line is additional evidence that the meniscus is torn; a negative result of the McMurray test does not rule out a tear McMurray testing was positive in only $57.1 \%$ of patient and effusion was noted in only $14.3 \%$ [17].

\subsection{The Grinding Test}

With the patient prone, the knee is flexed to 90 degrees and the anterior thigh is fixed against the examining table. The foot and leg are pulled upward to distract the joint and rotated to place rotational strain on the ligaments; When ligaments have been torn, this part of the test usually is painful. Next, with the knee in the same position, the foot and legs are pressed downward and rotated as the joint is slowly flexed and extended, when ameniscus has been torn, popping and pain localized to the joint line may be noted. Although the McMurray, Apleys and other tests cannot be considered diagnostic, they are useful enough to be included in the routine examination of the knee.

\subsection{The Squat Test}

Several repetitions of a full squat with the feet and legs alternately fully internally and externally rotated as the squat is performed. Pain usually is produced on either the medial or the lateral side of the knee, corresponding to the side of the torn meniscus. Pain in the internally rotated position suggest injury to the lateral meniscus, whereas pain in the external rotation suggests injury to the medial joint line or the lateral joint line, however, is a much more dependable localizing sign than the position of rotation.

\subsection{The Thessaly Test}

The examiner supports the patient by holding his or her outstretched hands while the patient stands flatfooted on the floor. The patient then rotates his or her knee and body, internally and externally, three times with the knee in slight flexion (5 degrees). The same procedure is carried out with the knee flexed 20 degrees. Patients with suspected meniscal tears experience medial or lateral joint-line discomfort and may have a sense of looking or catching. The test is 
always done on the normal knee first to teach the patient how to keep the knee in 5 and 20 degrees of flexion and how to recognize a possible positive result in the symptomatic knee. The Thessaly test at 20 degrees of knee flexion was suggested to be effective as a first-line clinical screening test for meniscal tears.

\section{Imaging}

With the improvements in CT [18] and MRI scanning [19], have been shown to improve diagnostic accuracy in many knee disorder. MRI scanning is first choice for diagnosis of meniscus tear rarely use of arthrograph.

A root tear could be demonstrated on pre-operative M.R.I in only $72.9 \%$ of the patient, while the rest demonstrated degeneration and/or fluid accumulation at the posterior horn without a visible meniscal tear [20]. Other, have reported significantly improved detection ability up to $93.3 \%$ sensitivity, $100 \%$ specificity and $100 \%$ positive predictive value using a variety of magnetic resonance sequences and interpretation signs suggestive of root tears [21] [22] [23]. The detailed for Meniscus tear and its management has been discussed in Figure 1 with a flowchart.

\section{Management of Meniscus Tear}

\subsection{Non-Operative Management}

If the knee is locked, it is manipulated under general anaesthesia. No special manoeuvre is needed. As the knee relaxes, the torn meniscus falls into place and knee is unlocked. The knee is immobilised in a Robert-Jones compression bandage for 2 - 3 weeks, followed by physiotherapy. In a case where locking is not present, Immobilisation with the Robert-Jones bandage is sufficient. With this, a small number of peripheral tear will heal. Rest of the tears may produce recurrent symptoms. Consists of a groin-to-ankle cylinder cast or knee immobilizer worn for 4 to 6 weeks. Crutch walking with touch-down weight bearing is permitted when the patient gains active control of the extremity in the cast. The patient is instructed in a progressive isometric exercise program during the time the legs is in the cast to strengthen the quadriceps, the hamstrings and the gastrocnemius and soleus muscles around the knee as well as the flexors, abductors, adductors and extensors around the hip. At 4 to 6 weeks, the immobilization is discontinued and the rehabilitative exercise program for the muscles around the hip and knee is intensified.

- Knee braces (Knee immobilizer): In most cases, the injury can heal within months. Surgery is rarely required only in most severe cases. Aside from surgery, less invasive treatments can help as well, including rest, ice, pain relievers and physical therapy. After a meniscus tear, patients are also going to start researching quality knee braces. Knee braces may help to recover from a torn meniscus injury and prevent aggravating and existing condition [24]. Let's delve into how a knee brace can help you recover from a torn meniscus. 


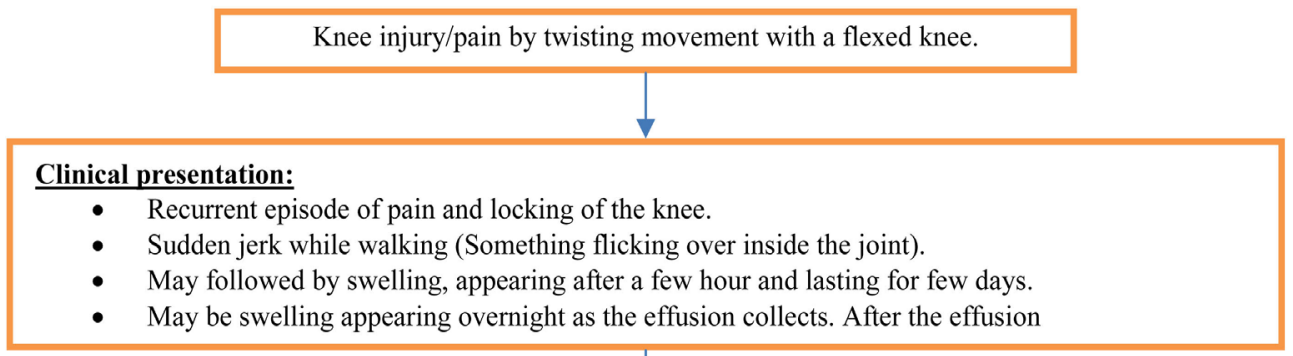

Chance to missing diagnosis of meniscal tears by clinical

Locking may be missed because the attention is drawn to more obvious signs of pain and swelling. The displaced fragment sometimes return to its original position spontaneously and thus the original episode of locking may never be noticed.

The history of sudden, locking and unlocking, with a click located in one or other joint compartment, is a diagnostic of meniscal tear.

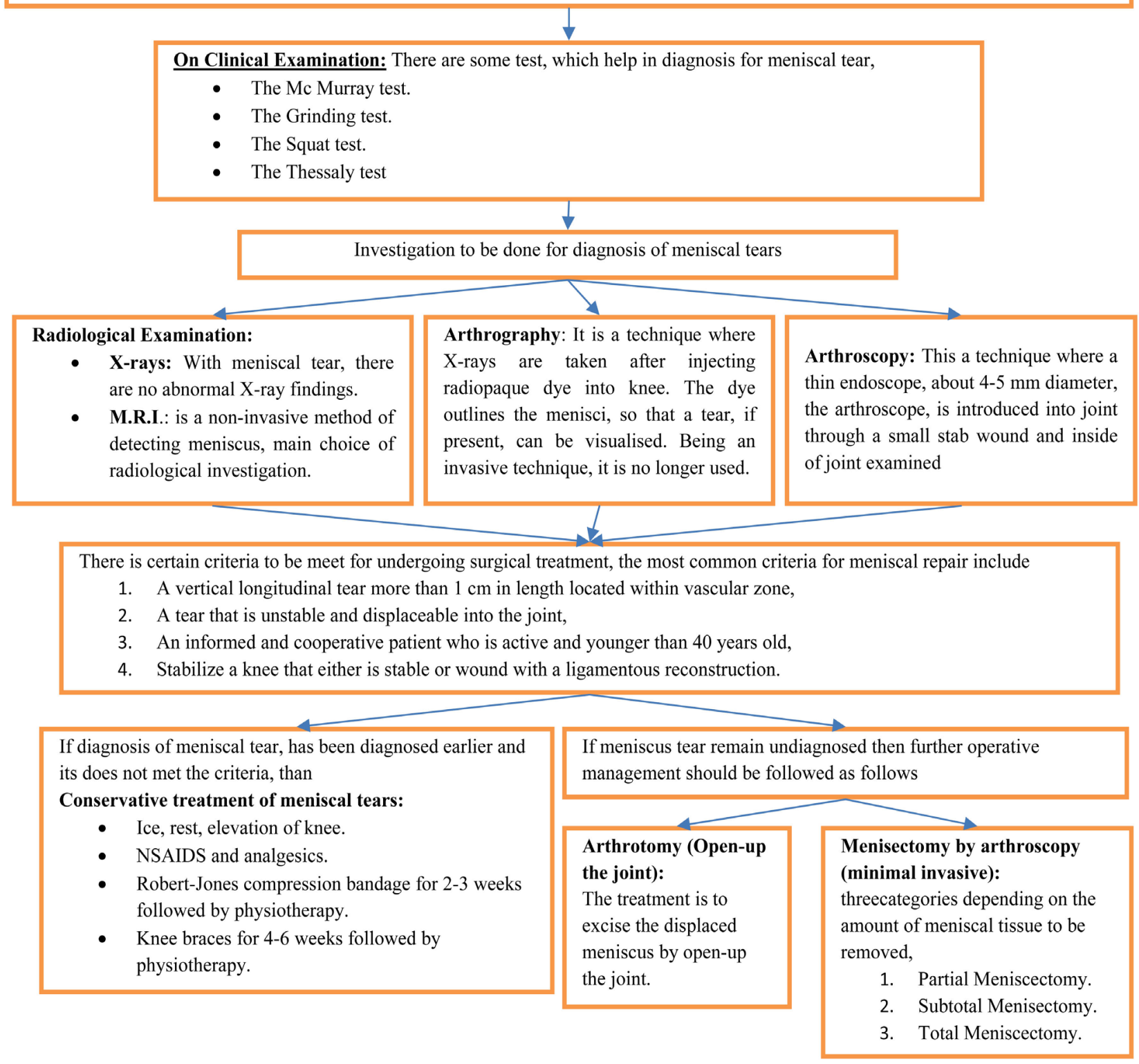

Figure 1. Flowchart on Meniscal tears and its management. 
o Providing stability. Wearing a rehabilitative knee brace will help the patient recover from a torn meniscus by providing stability to the joint and preventing the wearer from aggravating the injury even further, knee braces with hinges on either side of the knee offer protection and prevent that wobbly feeling patient may get after a knee injury.

o Preventing hyperextension: A knee brace will prevent the patient from extending the knee beyond their normal range of motion. One of the most common causes of a torn meniscus in hyperextension at the knee.

o Increased confidence: Wearing knee brace can help increase patient confidence and gie then the push they need to execute their therapy program and get batch on the road to recovery.

o Pain relief. A well-constructed knee brace will have an unloading effect on the wearer's knee. The brace reduces the direct pressure from patient upper leg on patient meniscus, which results in pain relief. When you have less pain, it is more likely that you will be more active and stick to your physical therapy program.

o Increased circulation: Knee braces add pressure to the affected area, which encourages greater circulation, Blood flow to the area brings oxygen and fresh nutrients which speed recovery. Research shows that about $30 \%$ of the meniscus (the outer portion) has its own blood supply. The rest of the meniscus receives its nutrition from synovial fluid in joint capsules. The nutrients in the synovial fluid diffuse to the meniscus through movement. Keep in mind one of the main benefits of knee braces is that they help you stay active, which is a major part of recovery.

- Exercise for meniscus tear and knee injury: The exercises increase in difficulty as pain levels decreases. Initially after the injury, you want the patient to rest, ice and elevate the knee, as well as wear a knee brace to help reduce swelling. Crutches may be advised for weight bearing activities such as walking. Soon after, depending on the damages, you want the patient to start working a range of motion. Exercise to help prevent further damage and further loss of range of motion. These exercises can be as easy as flexing and extending the knee, within pain free limits [25]. After the pain continues to decrease and the patient can perform range of motion exercise without pain, the patient can progress to the next stage of the rehabilitation process. The clinician can assist the patient in stretching the hamstrings, quadriceps and iliotibial band to help prevent the surrounding muscles from becoming tight. If the patient remains pain free, than simple exercise can be introduced [26].

o Quad sets exercise: With the injured leg fully extended, place a small towel under the back of the knee. Contract the Quadriceps muscles by pushing the knee towards the ground [27].

o Short-Arc-Quad-Extensions exercise: Place a towel under the injured knee, so the knee is bent at about $45^{\circ}$, extend the knee and held for about $10 \mathrm{sec}-$ onds than relax in the resting position. 
o Straight leg raises exercise: Lying flat on your back, bend your uninjured leg $90^{\circ}$, leaving your injured leg straight. Lift your injured leg about 12 inches off the ground, keeping your thigh muscles contracted and hold for about 5 seconds, then lower the leg slowly back of the ground.

o Toe raises (with Tubing) exercise: In a seated position with the injured leg fully extended, injured leg fully extended, tubing can be placed around the top of the foot (Either held by the clinician or tried to a table) and the athlete can perform toe raises with resistance.

o Lunges exercise: In a standing position, the patient brings one foot forwards and then bends at the knees keeping the back straight. The knee of the back leg should come close to the ground, and the knee of the front leg should not go past the toes, both legs should from a $90^{\circ}$ angle between the thigh and lower leg.

o Squats exercise: In a standing position, the patient should spread the feet to be above shoulder width apart. The patient should then band at knee to form a $90^{\circ}$ angle. The knee should not go past the toes, and the patient should make sure to maintain a flat back so as not to strain.

The patient must be informed that any tear in the meniscus may not have healed despite this period of immobilization [28]. If symptoms recur after a period of non-operative treatment, surgical repair or removal of the damaged meniscus may be necessary and more specific diagnostic procedures such as MRI and arthroscopy are used as indicated.

\subsection{Operative Management by Arthroscopy (Menisectomy)}

This is a technique where a thin endoscope, about $4-5 \mathrm{~mm}$ in diameter-the arthroscopy, is introduced into the joint through a small stab wound and inside of joint examined, repaired.

- General Principal for Arthroscopy: Arthroscopy of the knee can be done as the essential initial step before proceeding to operative arthroscopy or before an open arthrotomy. Anaesthesia can be local, regional block, or general. If the procedure is uncomplicated and of short duration, it can be done using local anaesthesia in cooperative patients, especially if the surgeon is experienced in arthroscopy [29]. If local anaesthesia is to be used, we prefer intravenous sedation for portal injection with $1 \%$ lidocaine and an intraarticular bolus of $30 \mathrm{ml}$ bupivacaine and $15 \mathrm{ml}$ of lidocaine 20 minutes before starting the procedure. Diagnostic arthroscopy before arthrotomy or major intraarticular surgery anesthesia, unless this type of anaesthesia is contraindicated. The procedure is performed in the operating room under strict sterile conditions. Must not minimized the seriousness of this surgical procedure. Although complications such as infection are infrequent $(<1 \%)$, carelessness in surgical scrubbing, preparation, or draping or careless handling of the irrigating solutions, arthroscopes, and instruments can result in intraarticular infection just as devasting as those after arthrotomy. Sterilization of arthro- 
scopy equipment and use of waterproof arthroscopy growns and drapes are essential. Sealing the extremity proximal and distal to the arthroscopy site and use of a durable skin preparation (Dura Prep) and iodine-impregnated drape at the surgical site can help to minimize infections.

The scrub nurse uses a large table for instruments. This is positioned for the nurse's convenience, usually on the same side as the knee having surgery. A Mayo stand is placed over the operating table at the upper part of the patient's thighs, and the more commonly used instruments are placed on it. Power cords and light cables are attached to the appropriate sources and are placed on a side table. Irrigation bags are suspended from an intravenous stand at the head of the table and are raised 3 to 4 feet above the level of the patient. The use of an arthroscopic pump for inflow through the arthroscope sheath or a separate sheath helps keep flows and pressure constant. The pump may eliminate the need for a tourniquet, making arthroscopy using local anaesthesia feasible. A tourniquet is placed around the thigh but is not inflated in diagnostic arthroscopy unless troublesome bleeding occur, Inflation of the tourniquet blanches the synovium and other vascularised tissues and make diagnostic evaluation of these structures more difficult. Meniscal vascularity and healing potential should be evaluated with the tourniquet deflated and the intraarticular hydrostatic pressure low. The tourniquet usually is inflated after exsanguinations of the limbs in acute traumatic disorders, or if the surgeon anticipates anything other than the simplest intaarticular surgical procedure. Tourniquet time should be minimized and not exceed 90 minutes for routine procedures to prevent possible deep vein thrombosis. For major complicated procedures, tourniquet times of 2 hours can be used, but times longer than this should be avoided to prevent ischemic neurovascular changes. Stressing the knee to open up the various compartments is necessary for diagnostic or operative procedures. This can be accomplished by using an assistant, a padded lateral post, or a commercial leg-holding device. The use of an assistant to stress the joint probably is the least efficient methods because of fatigue and the inconsistent amounts of stress that results, among other factors. The use of a padded lateral post attached to the edge of the operating table can be effective for valgus stressing in or near full extension, but it does not control rotation. The commercial thigh holders are most effective, but some of their potential dangers must be kept in mind. Although the use of leg-holding devices makes stressing and opening the compartments easier, especially the posterior compartment, these devices do get in the way when one is working through the superior portals in the patellofemoral joint. Also, the potential tourniquet effect of the leg-holding devices must be appreciated. In osteoporotic bone, excessive stress can result in fracture to the rigidly held extremity.

Once the diagnosis is established clinically, the treatment is it excise the displaced fragment of the meniscus by opening-up the joint (arthrotomy). Now a day, it is possible to excise a torn meniscus arthroscopically (arthroscopic surgery). By this technique, once the fault is detected e.g. a loose meniscal flap, the same is excised using fine cutting instruments introduced from another punc- 
ture wounds. This technique is a significant advance as it can be done as a day care procedure, sometimes even under local anaesthesia. Since it is a minimally invasive technique, early return to work is possible. Recent research has shown that menisci are not "useless" structure as was through earlier. Hence, wherever possible the trend is to preserve the meniscus by suturing (Meniscorraphy).

- General principles of meniscal excisions and different categories (Meniscectomy): Partial meniscectomy is always preferable to subtotal or total meniscectomy [30] [31]. Leaving an intact, balanced, peripheral rim of meniscus aids in the stability of joint and protects the articulars surface by its load-bearing function. Total meniscectomy removes all of the actual load-bearing protection and reduces stability of the joint, especially if a concomitant ligamentous relaxation already exists. Partial meniscectomy, although desirable, is not always possible if the tear extends to the periphery of the meniscus [32]. In such cases, subtotal excision is preferable to complete excision, even though the contoured anterior meniscal tissue left may be subject to sub-sequent tears or degeneration. To determine accurately the type of meniscectomy required, the meniscal lesion must be carefully probed and classified. Failure to classify, probe, and explore accurately and thoroughly the extent and various planes of tear before proceeding with the meniscal resection often results in needlessly sacrificing healthy meniscal tissues. When the meniscal tear has been probed and classified, the surgeon should mentally formulate the methods and steps required to excise the necessary portion of the meniscus. The surgeon should be able to visualize the tissues to be removed and the subsequent contour of the peripheral meniscal rim. The objectives is to remove the torn, mobile meniscal fragment and contour the peripheral rim, leaving a balanced, stable rim of meniscal tissues. Excision of the pathological tissue can be done either with en bloc resection of the mobile fragment or by morcellization of the fragments and subsequent removal. Sharp excision of the major mobile fragments usually is preferable to morcellization to minimize the potential debris within the joint [32]. When the tear has beenremoved, the remaining peripheral rim must be carefully probed to ensure that there are no additional tears and that the rim is balanced and stable. When a contoured, balanced, stable peripheral rim is present, the joint should be thoroughly lavaged and suctioned to remove any small meniscal fragments or debris that may have dropped into the joint as result of the resection.

- Types of meniscal excisions: O' Connor separated meniscal excisions into three categories depending on the amount of meniscal tissue to be removed.

o Partial Meniscectomy: In this type of meniscal excision, only the loose, unstable meniscal fragments are excised, such as the displaceable inner edge in bucket-handle tears, the flaps in flap tears, or the flaps in oblique tears. In partial meniscectomies, a stable and balanced peripheral rim of healthy meniscal tissues is preserved. 
o Subtotal Menisectomy: In this type of meniscectomy, the type and extent of tear require excision of a portion of the peripheral rim of the meniscus. This is the most commonly required in complex or degenerative tears of the posterior horn of either meniscus. Resection of the involved portion by necessity extends out to and includes the peripheral rim of the meniscus. It is termed subtotal because in most cases most of the anterior horn and a portion of the middle third of the meniscus are not resected.

o Total Meniscectomy: Total removal of the meniscus is required when it is detached from its peripheral meniscosynoial attachment, and intra-meniscal damage and tears are extensie. If the body of the peripherally detached meniscus is salvageable, total meniscectomy is not warranted and meniscal suture should be consider.

Admittedly, success rates arehigh [33] and complication rates low $(0.27 \%$ to $2.8 \%$ ). But Salzler [34] reported a complication rate of $2.8 \%$ and did not consider knee arthroscopy to be benign procedure. In contrast, since 2002 and more particularly 2013, several controlled randomized studied compared arthroscopic menisectomy versus non-operative management (mainly by physiotherapy) [35] [36] [37] [38] [39] or "sham" surgery [32] [40]. The older studies, by Moseley and Kirkley concerned osteoarthritis knee and others knee free of macroscopic oestparthritis. The meta-analysis by Thorlund and Kise's recent study confirmed these finding [41] [42]. However, it should be noted that cross-over for failure of non-operative treatment in the randomized studies, however well conducted, involved biases and limitation to which the reader must be alert [43] [44].

\section{Postoperative Care}

Toe-touch weight bearing with the use of crutches is allowed for $48 \mathrm{hr}$ until the patient is comfortable. Straight-leg raising exercise, ankle pumps, and range of motion exercise are started in recovery room and repeated hourly during the early postoperative period. Wall sets are started 3 to 4 days after surgery. Stationary bike and progressive low-impact strengthening exercise are started when postoperative swelling has resolved. Return to sport is allowed around 3 to 4 weeks.

\section{Discussion and Conclusion}

Many articles had been published regarding meniscal tears. After studying and analyzing all the methods and treatment of different articles, there is the possibility to cure by conservative treatment if patients get diagnosed earlier by different tests done by doctors in their clinical practises. Although the articles do not contain data and meta-analysis about this treatment, but have clearly focused on conservative treatment and advice of orthopaedic surgeon not to miss the meniscal tear during clinical practice. This review mainly aims on basic concept of meniscus tear and how to approach clinically and reach to proper diagnosis of meniscal tear, even experience of orthopaedic surgeon missed during 
clinical examination which further leads to chronic tears of meniscus. This review had shortly reviewed all concepts and management, and treatment on meniscus tear. Knee brace and knee exercise are quite helpful for meniscal tear patient and also are helpful to the knee injured patient. The injury can heal within months. Surgery is rarely required only in most severe cases. However, minimal invasive surgical procedure (Arthroscopy) is an advance treatment for chronic meniscus tear. It is very difficult to diagnose meniscus tear clinically without having any investigation, if the diagnosis is done by having minimum investigation and earlier than conservative treatment will have better results, which is quite economic to the patient, they need not to be admitted to hospital for as there is no further issues related to their health.

\section{Acknowledgements}

Prof. Lui Kebin (18972161001), liukb@sina.com, (H.O.D) Department of Orthopedics, the First Affiliated Peoples Hospital of Yangtze University, Jingzhou, China.

\section{Conflicts of Interest}

The authors declare no conflicts of interest regarding the publication of this paper.

\section{References}

[1] Ahn, J.H., Lee, Y.S., Yoo, J.C., Chang, M.J., Park, S.J. and Pae, Y.R. (2010) Results of Arthroscopic All-Inside Repair for Lateral Meniscus Root Tear in Patients Undergoing Concomitant Anterior Cruciate Ligament Reconstruction. Arthroscopy, 26, 67-75. https://doi.org/10.1016/j.arthro.2009.07.007

[2] Allaire, R., Muriuki, M., Gilbertson, L. and Harner, C. (2008) Biomechanical Consequences of a Tear of the Posterior Root of the Medial Meniscus. Similar to Total Meniscectomy. The Journal of Bone and Joint Surgery. American Volume, 90 1922-1931. https://doi.org/10.2106/JBJS.G.00748

[3] Johnson, D.L., Swenson, T.M., Livesay, G.A., Aizawa, H., et al. (1995) Insertion-Site Anatomy of the Human Menisci: Gross, Arthroscopic, and Topographical Anatomy as a Basis for Meniscal Transplantation. Arthroscopy, 11, 386-394. https://doi.org/10.1016/0749-8063(95)90188-4

[4] Jones, A.O., Houang, M.T.W., Low, R.S. and Wood, D.G. (2006) Medial Meniscus Posterior Root Attachment Injury and Degeneration: MRI Findings. Australasian Radiology, 50, 306-313. https://doi.org/10.1111/j.1440-1673.2006.01586.x

[5] Nicholas, S.J., Golant, A., Schachter, A.K. and Lee, S.J. (2009) A New Surgical Technique for Arthroscopic Repair of the Meniscus Root Tear. Knee Surgery, Sports Traumatology, Arthroscopy, 17, 1433-1436. https://doi.org/10.1007/s00167-009-0874-1

[6] Shepard, M.F., Hunter, D.M., Davies, M.R, Shapiro, M.S. and Seeger, L.L. (2002) The Clinical Significance of Anterior Horn Meniscal Tears Diagnosed on Magnetic Resonance Images. The American Journal of Sports Medicine, 30, 189-192. https://doi.org/10.1177/03635465020300020701 
[7] Fithian, D.C., Kelly, M.A. and Mow, V.C. (1990) Material Properties and Structure-Function Relationships in the Menisci. Clinical Orthopaedics and Related Research, 252, 19-31. https://doi.org/10.1097/00003086-199003000-00004

[8] Kohn, D. and Moreno, B. (1995) Meniscus Insertion Anatomy as a Basis for Meniscus Replacement: A Morphological Cadaveric Study. Arthroscopy, 11, 96-103. https://doi.org/10.1016/0749-8063(95)90095-0

[9] Kopf, S., Colvin, A.C., Muriuki, M., Zhang, X.D. and Harner, C.D. (2011) Meniscal Root Suturing Techniques: Implications for Root Fixation. The American Journal of Sports Medicine, 39, 2141-2146. https://doi.org/10.1177/0363546511413250

[10] Fox, A.J., Bedi, A. and Rodeo, S.A. (2012) The Basic Science of Human Knee Menisci: Structure, Composition, and Function. Sports Health, 4, 340-351.

https://doi.org/10.1177/1941738111429419

[11] Berlet, G.C. and Fowler, P.J. (1998) The Anterior Horn of the Medical Meniscus. An Anatomic Study of Its Insertion. The American Journal of Sports Medicine, 26, 540-543. https://doi.org/10.1177/03635465980260041201

[12] Nelson, E.W. and La Prade, R.F. (2000) The Anterior Intermeniscal Ligament of the Knee. An Anatomic Study. The American Journal of Sports Medicine, 28, 74-76. https://doi.org/10.1177/03635465000280012401

[13] Poh, S.Y., Yew, K.-S.A., Wong, P.-L.K., Koh, S.-B.J., Chia, S.-L., Fook-Chong, S., et al. (2012) Role of the Anterior Intermeniscal Ligament in Tibiofemoral Contact Mechanics during Axial Joint Loading. Knee, 19, 135-139.

https://doi.org/10.1016/j.knee.2010.12.008

[14] Zantop, T., Wellmann, M., Fu, F.H. and Petersen, W. (2008) Tunnel Positioning of Anteromedial and Posterolateral Bundles in Anatomic Anterior Cruciate Ligament Reconstruction: Anatomic and Radiographic Findings. The American Journal of Sports Medicine, 36, 65-72. https://doi.org/10.1177/0363546507308361

[15] Ziegler, C.G., Pietrini, S.D., Westerhaus, B.D., Anderson, C.J., Wijdicks, C.A., et al. (2011) Arthroscopically Pertinent Landmarks for Tunnel Positioning in Single-Bundle and Double-Bundle Anterior Cruciate Ligament Reconstructions. The American Journal of Sports Medicine, 39, 743-752. https://doi.org/10.1177/0363546510387511

[16] Pujol, N., Panarella, L., Selmi, T.A.S., Neyret, P., Fithian, D., et al. (2008) Meniscal Healing after Meniscal Repair: A CT Arthrography Assessment. The American Journal of Sports Medicine, 36, 1489-1495. https://doi.org/10.1177/0363546508316771

[17] Lee, J.H., et al. (2009) Arthroscopic Pullout Suture Repair of Posterior Root Tear of the Medial Meniscus: Radiographic and Clinical Results with a 2-Year Follow-Up. Arthroscopy, 25, 951-958. https://doi.org/10.1016/j.arthro.2009.03.018

[18] Pujol, N., Tardy, N., Boisrenoult, P. and Beaufils, P. (2015) Long-Term Outcomes of All-Inside Meniscal Repair. Knee Surgery, Sports Traumatology, Arthroscopy, 23, 219-224. https://doi.org/10.1016/j.arthro.2009.03.018

[19] Dujardin, D., Siboni, R., Kanagaratnam, L., Boisrenoult, P., Beaufils, P. and Pujol, N. (2017) Long-Term Assessment of Meniscal Extrusion after Meniscal Repair. Orthopaedics \& Traumatology: Surgery \& Research, 103, 373-376. https://doi.org/10.1016/j.otsr.2016.12.014

[20] Ozkoc, G., Circi, E., Gonc, U., Irgit, K., Pourbagher, A. and Tandogan, R.N. (2008) Radial Tears in the Root of the Posterior Horn of the Medial Meniscus. Knee Surgery, Sports Traumatology, Arthroscopy, 16, 849-854.

https://doi.org/10.1007/s00167-008-0569-z 
[21] Choi, S.H., Bae, S., Ji, S.K. and Chang, M.J. (2012) The MRI Findings of Meniscal Root Tear of the Medial Meniscus: Emphasis on Coronal, Sagittal and Axial Images. Knee Surgery, Sports Traumatology, Arthroscopy, 20, 2098-2103. https://doi.org/10.1007/s00167-011-1794-4

[22] De Smet, A.A., Blankenbaker, D.J., Kijowski, R., Graf, B.K. and Shinki, K. (2009) MR Diagnosis of Posterior Root Tears of the Lateral Meniscus Using Arthroscopy as the Reference Standard. American Journal of Roentgenology, 192, 480-486. https://doi.org/10.2214/AJR.08.1300

[23] Harper, K.W., Helms, C.A., Lambert, III, H.S. and Higgins, L.D. (2005) Radial Meniscal Tears: Significance, Incidence, and MR Appearance. American Journal of Roentgenology, 185, 1429-1434. https://doi.org/10.2214/AJR.04.1024

[24] Greenfield, J.R.J., Hwang, H.F., Davies, C. and McDaid, A.J. (2017) Soft-Stop Knee Brace for Rehabilitation from Ligament Injuries: Design and Pilot Trial. International Conference on Rehabilitation Robotics, London, 17-20 July 2017, 352-357. https://doi.org/10.1109/ICORR.2017.8009272

[25] Lowe, W.R., Warth, R., Davis, E. and Bailey, L. (2017) Functional Bracing after Anterior Cruciate Ligament Reconstruction: A Systematic Review. Journal of the American Academy of Orthopaedic Surgeons, 25, 239-249. https://doi.org/10.5435/JAAOS-D-15-00710

[26] Lu, T.W., Lin, H.C. and Hsu, H.C. (2006) Influence of Functional Bracing on the Kinetics of Anterior Cruciate Ligament-Injured Knees during Level Walking. Clinical Biomechanics, 21, 517-524. https://doi.org/10.1016/j.clinbiomech.2005.12.017

[27] Benedetti, A., Platt, R. and Atherton, J. (2014) Generalized Linear Mixed Models for Binary Data: Are Matching Results from Penalized Quasi-Likelihood and Numerical Integration Less Biased? PLoS ONE, 9, e84601. https://doi.org/10.1371/journal.pone.0084601

[28] Grotle, M. and Hagen, K.B. (2017) Exercise Therapy May Be as Effective as Arthroscopic Partial Meniscectomy in Treating Degenerative Meniscal Tears [Synopsis]. Journal of Physiotherapy, 63, 52. https://doi.org/10.1016/j.jphys.2016.10.009

[29] Whittington, C.F. and Carlson, C.A. (1991) Anterior Cruciate Ligament Injuries. Evaluation, Arthroscopic Reconstruction, and Rehabilitation. Nursing Clinics of North America, 26, 149-158.

[30] Sihvonen, R., Paavola, M., Malmivaara, A., Itälä, A., Joukainen, A., et al. (2018) Arthroscopic Partial Meniscectomy versus Placebo Surgery for a Degenerative Meniscus Tear: A 2-Year Follow-Up of the Randomised Controlled Trial. Annals of the Rheumatic Diseases, 77, 188-195. https://doi.org/10.1136/annrheumdis-2017-211172

[31] Azam, M. and Shenoy, R. (2016) The Role of Arthroscopic Partial Meniscectomy in the Management of Degenerative Meniscus Tears: A Review of the Recent Literature. The Open Orthopaedics Journal, 10, 797-804. https://doi.org/10.2174/1874325001610010797

[32] Sihvonen, R., Paavola, M., Malmivaara, A., Itälä, A., Joukainen, A., et al. (2013) Arthroscopic Partial Meniscectomy versus Sham Surgery for a Degenerative Meniscal Tear. The New England Journal of Medicine, 369, 2515-2524. https://doi.org/10.1056/NEJMoa1305189

[33] Khan, M., Evaniew, N., Bedi, A., Ayeni, O.R. and Bhandari, M. (2014) Arthroscopic Surgery for Degenerative Tears of the Meniscus: A Systematic Review and Meta-Analysis. Cmaj, 186, 1057-1064. https://doi.org/10.1503/cmaj.140433

[34] Salzler, M.J., Lin, A., Miller, C.D., Herold, S., Irrgang, J.J., et al. (2014) Complica- 
tions after Arthroscopic Knee Surgery. The American Journal of Sports Medicine, 42, 292-296. https://doi.org/10.1177/0363546513510677

[35] Kirkley, A., Birmingham, T.B., Litchfield, R.B., Giffin, J.R., Willits, K.R., et al. (2008) A Randomized Trial of Arthroscopic Surgery for Osteoarthritis of the Knee. The New England Journal of Medicine, 359, 1097-1107. https://doi.org/10.1056/NEJMoa0708333

[36] Herrlin, S.V., Wange, P.O., Lapidus, G., Hållander, M., Werner, S. and Weidenhielm, L. (2013) Is Arthroscopic Surgery Beneficial in Treating Non-Traumatic, Degenerative Medial Meniscal Tears? A Five Year Follow-Up. Knee Surgery, Sports Traumatology, Arthroscopy, 21, 358-364. https://doi.org/10.1007/s00167-012-1960-3

[37] Katz, J.N. and Losina, E. (2013) Surgery versus Physical Therapy for Meniscal Tear and Osteoarthritis. The New England Journal of Medicine, 369, 677-678. https://doi.org/10.1056/NEJMc1307177

[38] Yim, J.H., Seon, J.K., Song, E.-K., Choi, J.-I., Kim, M.-C., et al. (2013) A Comparative Study of Meniscectomy and Nonoperative Treatment for Degenerative Horizontal Tears of the Medial Meniscus. The American Journal of Sports Medicine, 41, 1565-1570. https://doi.org/10.1177/0363546513488518

[39] Gauffin, H., Tagesson, S., Meunier, A., Magnusson, H. and Kvist, J. (2014) Knee Arthroscopic Surgery Is Beneficial to Middle-Aged Patients with Meniscal Symptoms: A Prospective, Randomised, Single-Blinded Study. Osteoarthritis Cartilage, 22, 1808-1816. https://doi.org/10.1016/j.joca.2014.07.017

[40] Moseley, J.B., O’Malley, K., Petersen, N.J., Menke, T.J., Brody, B.A., et al. (2002) A Controlled Trial of Arthroscopic Surgery for Osteoarthritis of the Knee. The New England Journal of Medicine, 347, 81-88. https://doi.org/10.1056/NEJMoa013259

[41] Thorlund, J.B., Juhl, C.B., Roos, E.M. and Lohmander, L.S. (2015) Arthroscopic Surgery for Degenerative Knee: Systematic Review and Meta-Analysis of Benefits and Harms. BMJ, 350, h2747. https://doi.org/10.1136/bmj.h2747

[42] Kise, N.J., Risberg, M.A., Stensrud, S., Ranstam, J., Engebretsen, L., et al. (2016) Exercise Therapy versus Arthroscopic Partial Meniscectomy for Degenerative Meniscal Tear in Middle Aged Patients: Randomised Controlled Trial with Two Year Follow-Up. BMJ, 354, i3740. https://doi.org/10.1136/bmj.i3740

[43] Bollen, S.R. (2015) Is Arthroscopy of the Knee Completely Useless? Meta-Analysis-A Reviewer's Nightmare. The Bone \& Joint Journal, 97, 1591-1592. https://doi.org/10.1302/0301-620X.97B12.37456

[44] Elattrache, N., Lattermann, C., Hannon, M. and Cole, B. (2014) New England Journal of Medicine Article Evaluating the Usefulness of Meniscectomy Is Flawed. Arthroscopy, 30, 542-543. https://doi.org/10.1016/j.arthro.2014.02.010 